\title{
Editorial: Mobile and Ubiquitous Systems: Computing, Networking and Services
}

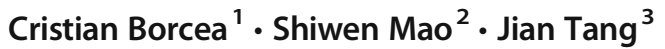 \\ Published online: 7 September 2019 \\ (C) Springer Science+Business Media, LLC, part of Springer Nature 2019
}

\section{Editorial:}

Computing, Networking and Services are important topics for existing and future mobile and ubiquitous systems, which have become a vibrant research field. This special issue contains extended versions of selected top papers from the proceedings of EAI MobiQuitous 2018 Conference, which aims to provide a forum for presenting and discussing top-notch quality research in the area of mobile and ubiquitous computing. MobiQuitous 2018 was held in New York City, NY, USA between 5 and 7 November, 2018. Over seventy researchers from all over the world attended the conference, which contained 11 technical sessions, a keynote presentation, a panel and a workshop. Through focused discussions, interaction and exchange of experiences, they fostered future research efforts and opened new leading directions for innovation and cutting-edge technologies.

This special issue features six high-quality articles. The first article, "Mining Regional Mobility Patterns for Urban Dynamic Analytics," proposes a region-aware mobility pattern mining framework, which jointly identifies the precise origin and destination region partitions while extracting mobility patterns. A Kernelized ACE scheme is proposed to solve the formulated optimization problem by learning feature representations that guarantee both objectives.

The second article is titled "Intelligent Human Counting through Environmental Sensing in Closed Indoor Settings," which addresses the problem of detecting count of human beings accurately in a closed indoor environment. The authors

Shiwen Mao

smao@ieee.org

1 Computer Science Department, New Jersey Institute of Technology, Newark, NJ, USA

2 Electrical and Computer Engineering, Auburn University, Auburn, AL, USA

3 Department of Electrical Engineering and Computer Science, Syracuse University, Syracuse, NY, USA

propose a novel methodology to count humans in closed indoor settings, which can be deployed in any hidden place being oblivious to intruders. The proposed scheme is based on different environmental gaseous parameters (Carbon Dioxide, Liquefied Petroleum Gas, Nitrogen Dioxide, and Sulfur Dioxide) and two weather parameters (temperature and humidity).

In the next article titled "Task Allocation in Eco-friendly Mobile Crowdsensing: Problems and Algorithms", the authors focus on the task allocation problem for eco-friendly mobile crowdsensing, aiming to minimize carbon emissions under various constraints such as task deadline and road traffic constraints. An offline Eco-Friendly Task Allocation algorithm (EFTA) and an online Eco-Friendly Task Allocation Algorithm (OEFTA) are proposed to solve the formulated problem.

Ride-on-demand (RoD) services use dynamic prices to balance the supply and demand to benefit both drivers and passengers, in an effort to improve service efficiency. The fourth article, "Fine-grained Dynamic Price Prediction in Ride-ondemand Services: Models and Evaluations," is focused on fine-grained dynamic price prediction, i.e., to predict the price for every single passenger request, while the prediction is performed by learning the relationship between dynamic prices and features extracted from multi-source urban data.

The fifth paper is titled "SmartMeeting: A Novel Mobile Voice Meeting Minutes Generation and Analysis System." It describes the design and implementation of SmartMeeting, an intelligent system for generating meeting minutes from meeting audio data. The authors also verify that that SmartMeeting can accurately summarize meetings and analyze agreed-upon actions by comparing and evaluating different baseline algorithms on real-world audio meeting datasets.

The last article, titled "Phascope: Fine-grained, Fast, Flexible Motion Profiling based on Phase Offset in Acoustic OFDM Signal," presents a study on exploiting acoustic Doppler shift estimation as a cost-effective way to implement Human-Computer Interaction applications across existing smart devices such as smart phones and smart speakers. 
Phase offset in acoustic OFDM signal is leveraged for developing Phascope, a fine-grained, fast and flexible motion profiling scheme.

Acknowledgements The guest editors would like to thank the Editor-inChief, Dr. Imrich Chlamtac for his support and guidance during the entire process. We are grateful to our EAI and Springer colleagues, Radka Pincakova, Rolissa Atienza, Eliska Vlckova, Leonora Panday, and Jed Joseph Adel; this special issue would not be possible without their help and support. We are also thankful to our reviewers for their effort in reviewing the manuscripts. Last but not least, we would like to thank all the authors for contributing their latest work to this special issue. S. Mao's research is supported in part by the NSF under grants CNS-1702957, ECCS-1923163, ECCS-1923717, and IIP-1822055. C. Borcea's research is supported in part by NSF grants CNS 1409523 and DGE 1565478, as well as by an ATT grant.

Publisher's Note Springer Nature remains neutral with regard to jurisdictional claims in published maps and institutional affiliations.

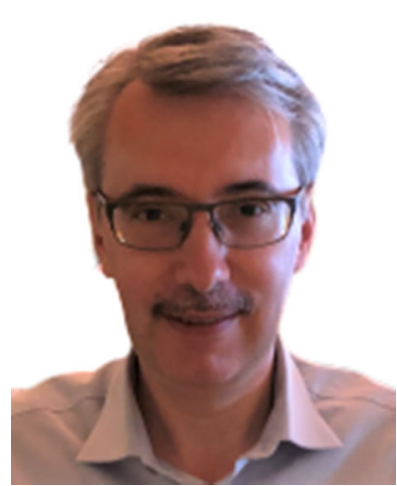

Cristian Borcea is a Professor in the Computer Science Department at New Jersey Institute of Technology, USA. He also holds a Visiting Professor appointment at National Institute of Informatics, Tokyo, Japan. Cristian's current research is on mobile cloud computing, edge computing, vehicular networking/computing, and machine learning for online advertising. Cristian received his PhD from Rutgers University, USA. He is a member of ACM

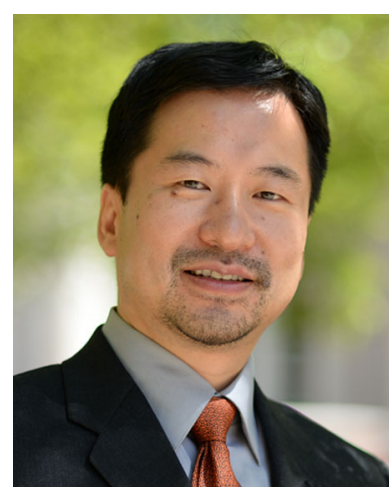

Shiwen Mao received a $\mathrm{PhD}$ in electrical and computer engineering from Polytechnic University, Brooklyn, N.Y., in 2004. He is the Samuel Ginn Professor and Director of Wireless Engineering Research and Education Center at Auburn University, Auburn, AL. His research interests include wireless networks, multimedia communications, and smart grid. $\mathrm{He}$ is a recipient of the Auburn University Creative Research \& Scholarship Award in 2018, the NSF CAREER Award in 2010 and The 2004 IEEE Communications Society Leonard G. Abraham Prize in the Field of Communications Systems. He is a Fellow of the IEEE.

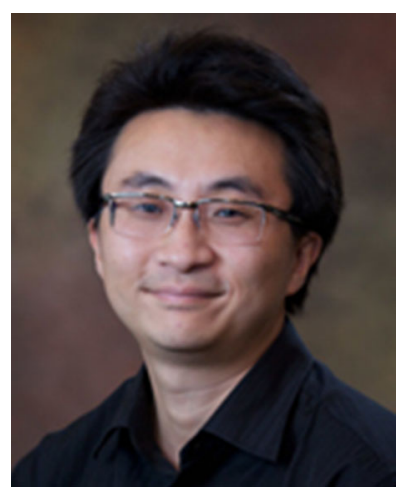

Jian Tang is a Professor in the Department of Electrical Engineering and Computer Science at Syracuse University and an IEEE Fellow. His research interests lie in the areas of Machine Learning, IoT, Wireless Networking and Big Data Systems. Dr. Tang has published over 140 papers in premier journals and conferences. He received an NSF CAREER award in 2009. He also received a few best paper awards, including IEEE ComSoc 2019 William R. Bennett Prize, IEEE VTS 2016 Best Vehicular Electronics Paper Award, Globecom'2015 and ICC'2014 Best Paper Awards, etc.

and IEEE. 